\title{
A Transient Spinal Cord Ischemia Reveals a Silent Type A Aortic Dissection
}

\author{
Rachelle Abi Nahed ${ }^{1 *}$ and Wissam Rizk ${ }^{2}$
}

${ }^{1}$ Department of Neurology, Faculty of Medicine, Hotel-Dieu de France Hospital, Université Saint-Joseph, Beirut, Lebanon

${ }^{2}$ Department of Neurology, Kesserwan Medical Center, Jounieh, Lebanon

Aortic dissection is a life-threatening vascular emergency that requires a prompt diagnosis and management. Isolated spinal transient ischemic accident is an extremely rare manifestation of aortic dissection (AD) type A. Thus, AD should be on the top of the aetiology list, despite the isolated character of the neurological presentation.

A 53-year-old man, previously healthy, presented to the emergency department for a right sided renal colic documented by a uroscan showing a right ureterovesical junction stone (Figure 1). Initial blood tests were normal. While symptomatic treatment was being administered, he presented an acute paraplegia with anaesthesia of both lower limbs in all modalities below T4. Deep tendon reflexes were absent and plantar reflex was indifferent bilaterally. His blood pressure

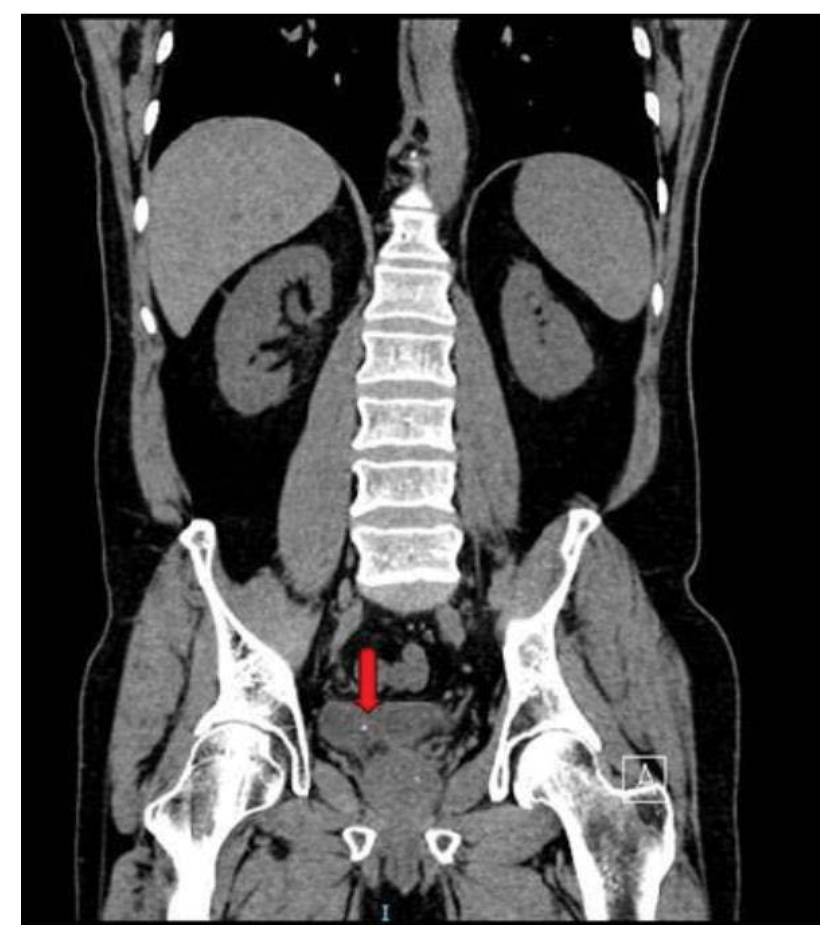

Figure 1: Helical CT of the abdomen and pelvis without intravenous or oral contrast administration, showing a $1.8 \mathrm{~mm}$ right ureterovesical junction calculus (arrow). was 200/140 mmHg. His symptoms self-resolved after 20 minutes. He denied any significant thoracic or back pain, and had no signs of limb ischemia.

Cardiac auscultation was normal and pulses were palpable and symmetric in all extremities.

Electrocardiogram, transthoracic echocardiography and enhanced total spine MRI (Figure 2) were all normal. CT angiography of the aorta (Figure 3) identified a Stanford type A $A D$ extending from the supravalvular segment until the-right iliac artery. All branches of the aorta arose from the true lumen. The patient's blood pressure was controlled with intravenous labetalol and he received urgent surgical repair of the dissection with a $36 \mathrm{~mm}$ Hemashield graft and an aortic valve replacement with a 25 St. Jude Trifecta valve.

His evolution was uneventful and was discharged home soon after.

The transient ischemic attack of the spinal cord is a very rare neurologic condition with an unknown prevalence. It constitutes $1 \%$ - $2 \%$ of all neurologic vascular pathologies [1].

$A D$ is also a rare occurrence. It has an incidence of 5 to 30 cases per million people per year, with the most typical symptoms being chest or back pain [2]. The symptoms and signs depend upon the extent of the dissection and the affected cardiovascular structures.

However, neurological symptoms can occur because of occlusion of carotid, vertebral, spinal arteries, vasa nervorum of peripheral nerves, or because of hypotension and related cerebral perfusion deficit [3]. They may be dramatic, and are sometimes the only symptoms, masking the underlying dis-

\footnotetext{
*Corresponding author: Rachelle Abi Nahed, Department of Neurology, Faculty of Medicine, Hotel-Dieu de France Hospital, Université Saint-Joseph, Beirut, Lebanon
}

Accepted: May 06, 2021

Published online: May 08, 2021

Citation: Nahed RA, Rizk W (2021) A Transient Spinal Cord Ischemia Reveals a Silent Type A Aortic Dissection. J Neurosurg Res Rev 4(1):40-42

Copyright: (c) 2021 Nahed RA, et al. This is an open-access article distributed under the terms of the Creative Commons Attribution License, which permits unrestricted use, distribution, and reproduction in any medium, provided the original author and source are credited. 


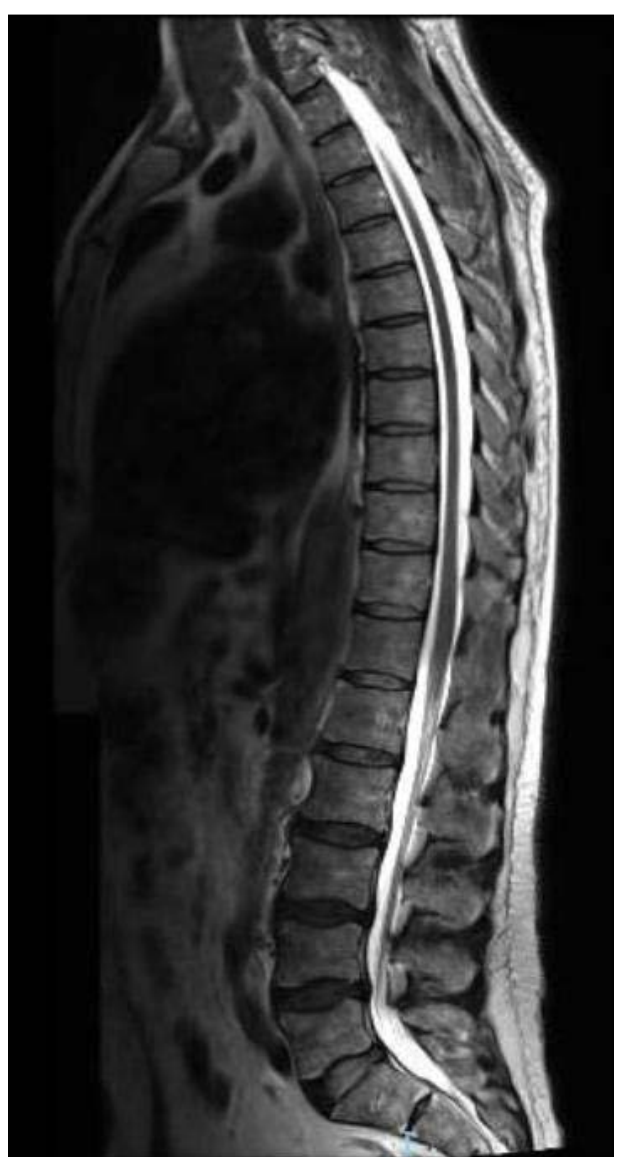

Figure 2: Total spine 3 Tesla MRI, with a T2-weighted sagittal plane shown here, with normal thickness and signal intensity of the spinal cord and conus medullaris. ease [4]. It is especially in these pain-free dissections (which occurs in 5-15\%) [5] with predominant neurological symptoms that the diagnosis of AD is difficult and delayed, and therefore increasing the mortality rate. Neurological presentation in painless type A AD include ischemic stroke (15.7\%), ischemic neuropathy $(10.8 \%)$, syncope $(5.9 \%)$, hypoxic encephalopathy (2\%) and spinal cord ischemia (1\%) [3]. The latter has been reported in few cases as the presentation of mostly type B AD. It can manifest as a complete transverse spinal cord infarction as well as anterior spinal cord syndrome, Brown-Sequard syndrome, progressive myelopathy or more rarely transient spinal cord ischemia [6-8].

We report the first case of a spinal transient ischemic accident as the only manifestation of a type $A$ aortic dissection.

Therefore, it is important to maintain a high-index of clinical suspicion for aortic dissection even if neurological symptoms are fluctuating or have fully remitted, in order to hasten the diagnosis and the adequate treatment.

\section{References}

1. Vargas MI, Gariani J, Sztajzel R, et al. (2015) Spinal cord ischemia: Practical imaging tips, pearls, and pitfalls. AJNR Am J Neuroradiol 36: 825-830.

2. Khan IA, Nair CK (2002) Clinical, diagnostic, and management perspectives of aortic dissection. Chest 122: 311-328.

3. Gaul C, Dietrich W, Friedrich I, et al. (2007) Neurological symptoms in type A aortic dissections. Stroke 38: 292-297.

4. Lee SJ, Kim JH, Na CY, et al. (2013) Eleven years of experience with the neurologic complications in Korean patients with acute aortic dissection: A retrospective study. BMC Neurol 13: 46.

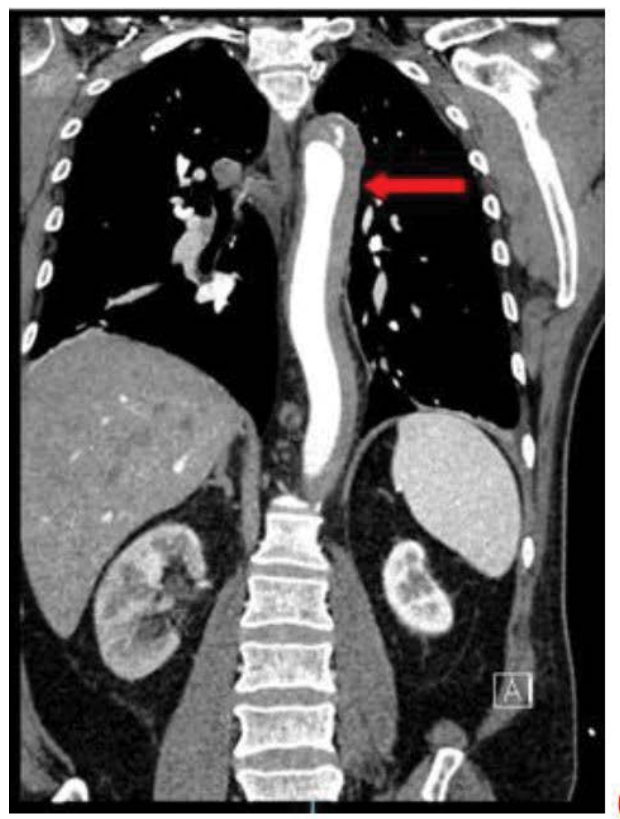

(A)

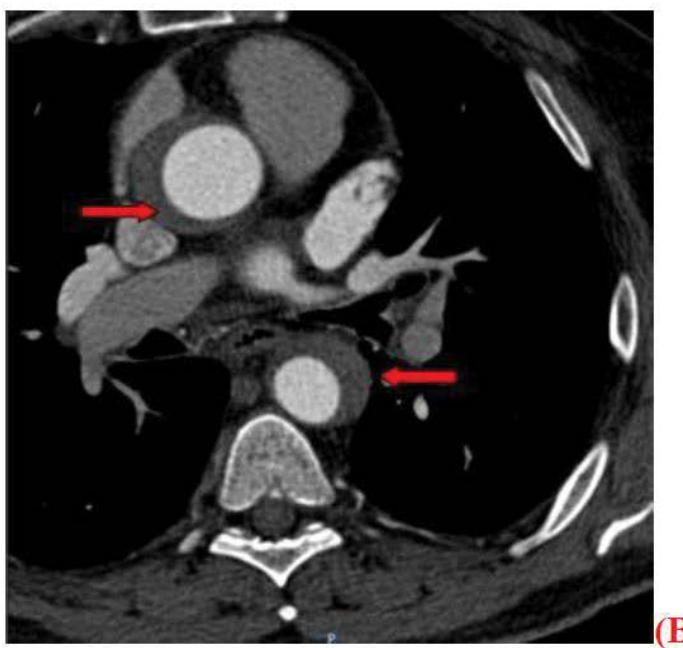

B)

Figure 3: $\mathrm{CT}$ angiography of the thoracic and abdominal aorta with a coronal A) and axial; B) plain, showing a Stanford type A aortic dissection extending from the supravalvular segment and of the thoracic aorta and reaching the aortic bifurcation (arrow), with almost complete thrombosis of the false lumen at the thoracic level and with patent false and true lumens at the abdominal juxtarenal and infrarenal level. 
5. Gaul C, Dietrich W, Erbguth FJ (2008) Neurological symptoms in aortic dissection: A challenge for neurologists. Cerebrovasc Dis Basel Switz 26: 1-8.

6. Beggs AD, Al-Rawi H, Parfitt A (2005) Chest pain and fleeting neurological signs. Lancet Lond Engl 365: 1514.
7. Colak N, Nazli Y, Alpay MF, et al. (2012) Aortic dissection presenting as paraplegia. Tex Heart Inst J 39: 273-276.

8. Gerber O, Heyer EJ, Vieux U (1986) Painless dissections of the aorta presenting as acute neurologic syndromes. Stroke 17: 644647. 